\title{
ICALP - International Center for Animal Law and Policy
}

Keywords: Icalp; Derecho Animal; Bienestar Animal; Políticas públicas

El ICALP es un Centro de Investigación y Enseñanza (CER), una estructura creada y prevista por la Universitat Autònoma de Barcelona (UAB) en su normativa, para fomentar la investigación y la enseñanza transversal.

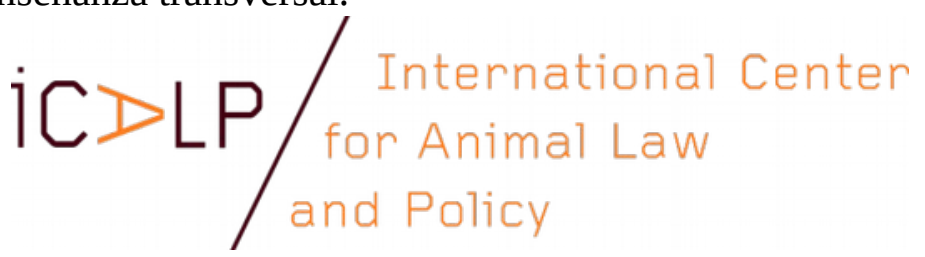

Como tal fue aprobado por el Consell de Govern de la UAB en su sesión de 11 de noviembre de 2015, pero no ha sido hasta recientemente que, por distintas vicisitudes académicas, ha podido celebrarse la sesión constitutiva de todos sus miembros, el día 11 de mayo de 2016. Hemos esperado pacientemente, hasta que todos los extremos de la organización han estado perfectamente integrados. No tenemos prisa, pero sí urgencia de que nuestro trabajo redunde en beneficio del diálogo científico que fomentarán los estudios centrados en la relación humanos-animales.

Existen prestigiosos Institutos y

Centros de Investigación

especializados en Ciencias del

Bienestar Animal (IRSEA, Institut de

Recherche en Sémiochimie et

Ethollogie Apliquée (Avignon,

Francia), Istituto G. Caporale (Teramo, Italia). Existen centros de Estudios de Derecho Animal, especialmente en: USA (ALDF, CALS. Lewis and Clark University. Center for Animal Law Studies. Portland, USA, Michigan State University College of Law, Animal

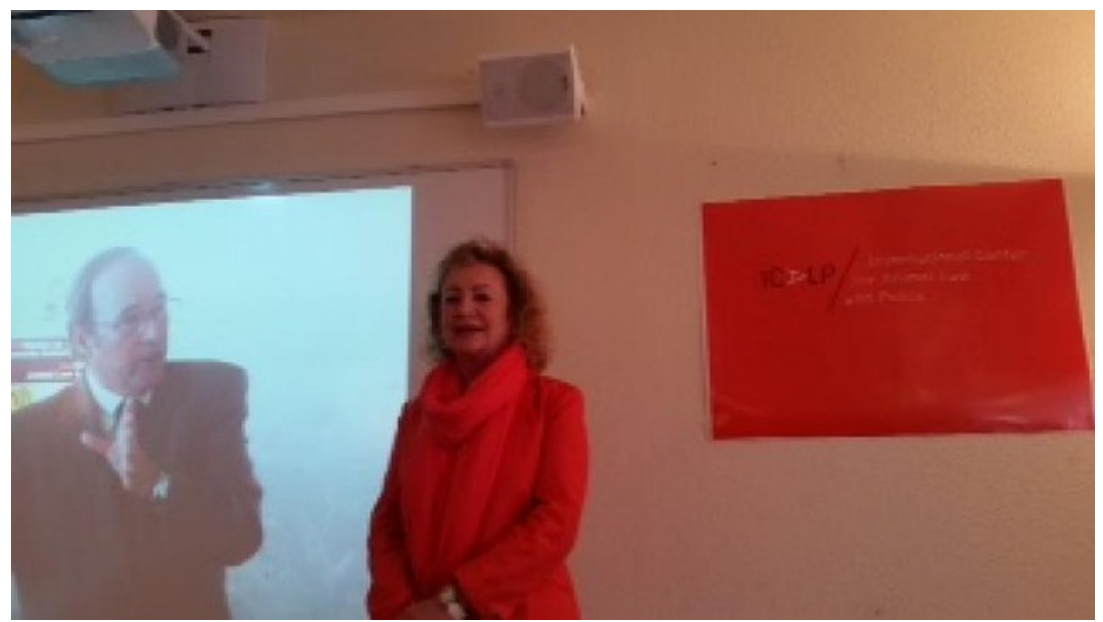
Law Web Center. Michigan, USA);

Australia (Minding Animals); Canadá (GRIDA). La promoción de Políticas Públicas de protección animal son la base de la existencia de antiguas y prestigiosas organizaciones animalistas en todo el mundo: Humane Society International (USA), Compassion in World Farming (UK); 30 Millions d'Amis (France), Vier-Pfoten (Austria).

Con todas las instituciones aquí referenciadas, así como con otras que sería prolijo detallar, hemos trabajado previamente y establecido contactos, tanto docentes, de investigación y de intercambio de estudiantes en prácticas, canalizadas desde el SGR Grupo de Investigación ADS-UAB.

Tenemos constancia -y así se visualiza en las cartas de apoyo que hemos ido recibiendo, de que la comunidad científica internacional, está mostrando su apoyo e interés en la creación del ICALP, pues en Europa, a día de hoy, no existe una iniciativa semejante. 


\section{Misión}

La Ciencia del Bienestar Animal, ha cumplido acabadamente -durante muchos años- el papel de investigar las necesidades de los Animales como "seres sintientes", expresión ésta procedente del mundo de la Ciencias relativas a los Animales y el medio ambiente, que se ha integrado dentro del mundo jurídico como expresión del grado de tratamiento y protección de los Animales, que el sistema jurídico ya ha hecho suya. Hoy en día, tanto en el campo científico como en el jurídico y social, la expresión "sentient beings" constituye tanto una frontera para rechazar y castigar aquellas prácticas que constituyen maltrato animal, como el punto de partida para construir recursos jurídicos y políticas públicas de protección de los intereses de los animales dentro del medio que les es propio, por lo que, la extensión de esta protección al entorno donde la mayoría de los Animales viven, que se suele reconocer como protección de la Biodiversidad, resultan intereses concomitantes.

Bien es cierto, que la confluencia de los intereses de las ciencias implicadas en la consecución de un objetivo constituye un fenómeno "natural". En el sentido de que las ciencias, cuando lo son, no son minúsculos espacios de conocimiento encerrados en sí mismos, sino, muy al contrario, tienden a la especialización abierta y a la cooperación entre profesionales que puedan aportar sus conocimientos para reforzar y avanzar en los propios hallazgos.

El tratamiento de los Animales se contempla a día de hoy como un espacio holístico de estudio y acción, pero no hay demasiados ejemplos de verdadera cooperación científica organizada, salvo el que, desde hace años venimos practicando en la UAB y esfera UAB algunos profesionales del Derecho, Veterinaria, Ciencias Clásicas, Ciencias de la Salud, Sociología, Biodiversidad. De ahí la coherencia de la propuesta de creación del ICALP (International Center for Animal Law and Policy).

No existe ningún centro reconocido internacionalmente que aúne los ámbitos que nosotros hemos venido promoviendo hasta ahora, de una forma dinámica e integrada. De ahí el interés y singularidad de nuestra propuesta de un Centro, que pueda cubrir esta necesidad dentro del campo internacional y posicione a la UAB -como lo ha sido hasta ahora-, como una Universidad pionera en los estudios de Derecho Animal y Políticas públicas.

El ICALP será el primer Centro interdisciplinario, interdepartamental e internacional en España, que integre estructuralmente las Ciencias Sociales, las Humanidades, las Ciencias de la Salud y las Ciencias del Bienestar Animal. Pensamos que este hecho producirá una sinergia importante entre cuatro grandes comunidades científicas tradicionalmente separadas, que, mediante nuestra propuesta, se involucran de forma integral en todos los aspectos relativos a los animales.

\subsection{Retos}

Entre los retos que creemos que estamos en disposición de asumir, se detallan a continuación los siguientes:

- Desde el punto de vista del Derecho, se podría esperar de la aplicación de la sentiencia de los animales, una mejora de la legislación tanto constitucional, civil como administrativa y penal muy significativa.

- Desde el punto de vista social, la promoción de una conciencia ciudadana informada y objetiva. Ello demanda la adaptación de los usos, que caracterizan a una sociedad que ha integrado a los animales como seres dignos de respeto, dentro de la vida diaria: transportes, alojamiento, vacunación, identificación, consumo (información, etiquetaje, responsabilidad), educación en el respeto en todos los niveles de enseñanza, turismo responsable, relación humanoanimal desde la perspectiva de la tenencia responsable y la promoción de vínculos estables, la manipulación de los animales, la definición de las necesidades de bienestar físico y comportamentales de los animales, las condiciones y límites del estudio de los animales y de los tests que se puedan realizar, la obligación de aliviar el dolor, el estrés, el miedo y la enfermedad de los animales, establecer penas graves para los casos de abuso y maltrato de animales y la violencia 
contra ellos y contra los elementos más vulnerables de la sociedad, niños especialmente.

- Desde el punto de vista de la agenda pública, la

implementación de las políticas tendentes a proteger el interés del animal de no ser maltratado -lo que hoy es un postulado reconocido por la ciencia del Bienestar Animal- , con el mismo nivel de interés que tiene el titular de la propiedad de proteger su derecho.
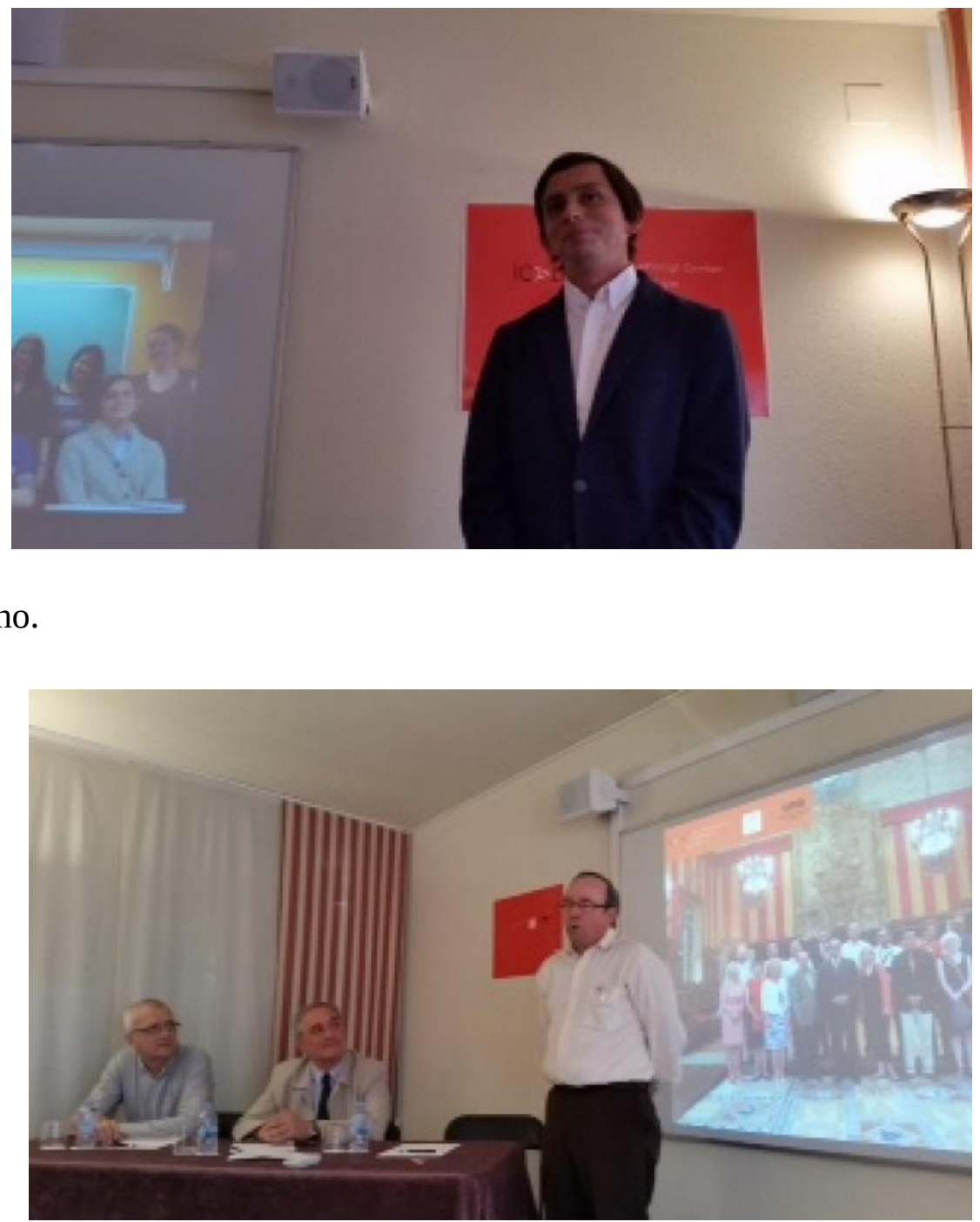

Afrontaremos estos objetivos esenciales desde las tres vertientes inherentes a la actividad universitaria: la investigación de calidad (como SGR reconocido), la docencia a través de los Máster en Derecho Animal y Sociedad (presencial y online) y la transferencia de resultados, como fundadores y miembros de EGALS de EUROPEAN LAWYERS FOR ANIMAL WELFARE y del EUROGROUP FOR ANIMALS, con los que ya hemos colaborado en la redacción de un informe europeo sobre equinos.

\subsection{Estructura}

La división estructural del ICALP tomará como base los dominios temáticos en aquellas áreas convergentes a la propuesta, que se estructurarán de manera que, siempre que sea posible, pueda favorecer la incorporación de nuevos miembros de las diferentes áreas de conocimiento:

- Derecho Animal

- Ciencia del Bienestar Animal

- Humanismo e Interacción Social

- Biodiversidad y Ciencias de la Salud

- Plataformas Multimedia y Políticas Públicas

\section{Objetivos de investigación}

Como objetivos de este Centro de Investigación, que es, por su misma composición multidisciplinar e internacional, destaca:

- Lograr una definición jurídica del animal, que lo distinga de la consideración de "cosa" o "producto"'

- Posicionar el bienestar animal como un compromiso dentro del desarrollo sostenible-. Contribuir, 
normativamente, a regular el bienestar de los animales (de granja, de experimentación, de espectáculos)

- Promover estudios sectoriales destinados a explorar la relación humanos-animales en ámbitos transversales: jurídico, social, económico, cultural, salud, alimentación, biodiversidad

- Aunar esfuerzos de los agentes potencialmente implicados en el bienestar animal

- Incidir en la masa crítica de los estudios relativos al trinomio Animales, Derecho y Políticas a través de publicaciones

- Obtener recursos a través de proyectos competitivos y posicionar los estudios realizados por el ICALP en un nivel de impacto y de calidad científica internacional.

- Organizar reuniones científicas, congresos y foros de opinión para atraer a la UAB a especialistas del máximo nivel, en los temas prioritarios del ICALP.

- Posicionar a la UAB como Universidad pionera en tema de estudios sobre Animales, Derecho y Sociedad.

- Diseminar los resultados de la investigación a través de la formación de profesionales, por medio de Masters, Cursos de Formación continua, Seminarios y workshops.

\section{Miembros y función}

El ICALP agrupa un núcleo significativo de investigadores:

- 12 investigadores de la UAB; 14 de Universidades extranjeras, entre los que se cuentan reconocidos expertos de nivel mundial; 11 profesionales, de reconocido prestigio en el ámbito que se propone. Con todos los miembros se vienen desarrollando proyectos y participación en actividades de investigación, además de las dedicadas a la enseñanza.

- Presenta el grupo del ICALP un volumen significativo de actividad, concentrada en los años 2005 a 2015, especialmente a partir de la formación del SGR Grup de Recerca ADS (UAB) y de la actividad desarrollada en el Curso de Postgrado "Animales, Derecho y Sociedad" (6 $6^{\text {a }}$ edición) y en el Master in Animal Law and Society ( $5^{\mathrm{a}}$ edición) y en el Master online en Derecho Animal y Sociedad ( $2^{\mathrm{a}}$ edición).

- La experiencia del Grupo de Investigación ADS ha sido hasta el momento muy satisfactoria. Sobre todo teniendo en cuenta que se trata de un campo emergente dentro del Derecho. En este sentido, se han obtenido proyectos y ayudas en forma de convenios, así como reconocimientos, participación y fundación de Grupos Internacionales de Estudio, publicaciones, Congresos, Workshops y experiencia editorial. Hemos incorporado las publicaciones y Proyectos de los últimos años de algunos de los miembros participantes, como muestra de la intensa actividad que se ha llevado a cabo y que augura el nivel de rendimiento y competividad que esperamos lograr con la creación de estenuevo Centro denominado ICALP.

\section{1.}

El ICALP será el primer CER interdisciplinario, interdepartamental e internacional que integre estructuralmente las Ciencias Sociales, las Humanidades, las Ciencias de la Salud y las Ciencias del Bienestar Animal. Pensamos que este hecho producirá una sinergia importante entre cuatro grandes comunidades científicas tradicionalmente separadas, que, mediante nuestra propuesta, se involucran de forma integral en todos los aspectos relativos a los animales.

Las Ciencias de la Antigüedad atesoran una inmensa riqueza de materiales y reflexiones que hay que seguir explorando, que explican la concepción social de los animales en el mundo occidental, la creación de los parámetros de la salud y de los remedios para conservarla dentro del mundo Mediterráneo, la conexión de los ritos religiosos con la animalidad, el respeto por la naturaleza y la conexión cosmológica con la misma, la función económica de los animales, que apenas sufrió variaciones en el mundo civilizado hasta el S. XIX. Todo ello, es el marco de pensamiento que abona en el mundo romano la calificación jurídica de los animales como cosas en propiedad. Por tanto, en el CER que se propone es una aportación muy valiosa y poco explorada. 
La discusión de la situación jurídica de los animales en el Derecho es a menudo infravalorada en el ámbito jurídico. Sin embargo, hay una abundantísima literatura específica sobre los cambios operados en el Código Civil en Alemania y en Austria en 1988 y 1990, que cambiaron el estatus de los animales de una simple objeto a una categoría en particular entre el ser humano y las cosas, la denominación adoptada fue la de "no cosas".

Uno de las metas de la creación del ICALP, es precisamente,

1. introducir la discusión científica sobre el estatuto jurídico de los animales al nivel de debate jurídico que le corresponde y que se está ya llevando a cabo en muchos países.

2. Desde nuestro punto de vista es muy importante saber si el animal es tratado como un simple objeto en la legislación o puede tener un estatus propio y diferenciado, como recientemente le ha otorgado Francia en el Code civil. De dicha actualización no se seguirían sólo beneficios para los animales, sino para la ciencia jurídica, que abriría una nueva frontera de debate, con repercusiones para la sociedad en su conjunto y para la solución de algunos problemas concretos que las Ciencias del Bienestar Animal han ido ya resolviendo, como, p.e., la situación de los animales de granja, de compañía o de espectáculos, que siguen esperando una solución a nivel global. Por ello, a día de hoy, se producen paradojas como que, dentro de la sociedad, el animal no se considera como un simple objeto. Es decir, se admite que a menudo son vistos como miembros en una familia, la carne de los animales se come pero la preocupación de cómo se ha producido todavía no constituye una cuestión de interés prioritario para el consumidor medio. Pensamos que reconocer un nuevo estatuto jurídico de los animales en el Derecho, puede llevar a una mejor comprensión de la propia sociedad en la que vivimos.

En consecuencia, cuando pensamos en seres sentientes, la ciencia del Bienestar Animal no excluye a ningún animal. La sentiencia animal y el reconocimiento de su protección está en relación, la mayoría de las veces, con el rendimiento económico que de los animales se obtiene, pero el respeto por los animales y por sus intereses -lo que constituye el núcleo del Derecho Animal-, no tiene, de entrada, límites por el tamaño, la belleza, el uso que se les dé, o por la proximidad mayor o menor con nuestra vida cotidiana. Otra cuestión es cómo debería articularse hoy en día ese triple aspecto:

1. El respeto social por los animales,

2. Las políticas públicas para hacerlo efectivo y

3. La protección jurídica de los animales como seres sentientes, capaces de experimentar dolor y placer, miedo o estrés.

Las Ciencias de la Salud, en tema animal, tienen un importante rol que cumplir en relación a dos cuestiones, que nos aparecen como prioritarias: las bases científicas de una nutrición saludable y el aprovechamiento sostenible, así como la relación humanos-animales desde el punto de vista de la infancia y la violencia. En este sentido hemos potenciado desde el principio la relación con el CST, que tiene amplia experiencia y dedicación a estos temas y colaboran en la investigación y en la docencia del Máster desde sus inicios.

Creemos que esta combinación de intereses científicos será muy positiva, porque nos permitirán ser globalmente más competitivos y productivos en la investigación básica y aplicada: publicación de trabajos científicos, generación y protección del conocimiento, programa de Doctorado Internacional de alta calidad (dentro de la Escuela de Doctorado de la UAB), fomento de alta especialización en los Masters de Derecho Animal existentes (presencial y online), transferencia del conocimiento a la sociedad, participación en proyectos internacionales, movilidad internacional de los investigadores, del personal en formación y de los estudiantes en prácticas. 